\title{
3-Dimensional Microorifice Fabricated Utilizing Single Undercut Etching Process for Producing Ultrasmall Water and Chitosan Droplets
}

\author{
Che-Hsin Lin, ${ }^{1}$ Chun-Hung Lan, ${ }^{1}$ and Chih-Hui Yang ${ }^{2}$ \\ ${ }^{1}$ Department of Mechanical and Electromechanical Engineering, National Sun Yat-Sen University, Kaohsiung 804, Taiwan \\ ${ }^{2}$ Department of Biological Science and Technology, I-Shou University, Kaohsiung 824, Taiwan \\ Correspondence should be addressed to Che-Hsin Lin; chehsin@mail.nsysu.edu.tw
}

Received 10 September 2013; Accepted 5 October 2013

Academic Editor: Liang-Wen Ji

Copyright (C) 2013 Che-Hsin Lin et al. This is an open access article distributed under the Creative Commons Attribution License, which permits unrestricted use, distribution, and reproduction in any medium, provided the original work is properly cited.

\begin{abstract}
This research reports a microfluidic device for producing small droplets via a microorifice and a T-junction structure. The orifice is fabricated using an isotropic undercut etching process of amorphous glass materials. Since the equivalent hydraulic diameter of the produced microorifice can be as small as $1.1 \mu \mathrm{m}$, the microdevice can easily produce droplets of the size smaller than $10 \mu \mathrm{m}$ in diameter. In addition, a permanent hydrophobic coating technique is also applied to modify the main channel to be hydrophobic to enhance the formation of water-based droplets. Experimental results show that the developed microfluidic chip with the ultrasmall orifice can steadily produce water-in-oil droplets with different sizes. Uniform water-in-oil droplets with the size from $60 \mu \mathrm{m}$ to $6.5 \mu \mathrm{m}$ in diameter can be formed by adjusting the flow rate ratio of the continuous phase and the disperse phases from 1 to 7 . Moreover, curable linear polymer of chitosan droplets with the size smaller than $100 \mu \mathrm{m}$ can also be successfully produced using the developed microchip device. The microfluidic T-junction with a micro-orifice developed in the present study provides a simple yet efficient way to produce various droplets of different sizes.
\end{abstract}

\section{Introduction}

Droplets of the size in micrometer or nanometer for advanced pharmaceutical and biomedical applications have become a popular research field in the recent years [1]. The production of medicine embedded microemulsion droplets is one of the important topics in this research field [2]. Dropletbased microfluidic devices have played important roles in the formation of various functional droplets in a highthroughput and high precision way [3]. A number of so-called "emulsification" techniques including T-junction shearing [4-7], flow focus necking [8, 9], and pneumatic pumping [10] have been reported to generate droplets in a continuous manner.

Over the reported microfluidic devices for droplets formation, micro-T-junction generator is the most popular scheme for producing droplets in devices. This droplet formation technique relies on the inherence properties of the employed liquids. T-junction microchannel devices provide a sharp edge where the continuous liquid phase cuts the dispersed liquid phase into small droplets by shearing force. The surfactant between the two immiscible liquids prevents the combination of these two liquids. Droplets with the size from tens to hundreds micrometers can be easily formed with this simple microfluidic structure. In general, the operation of this kind of microfluidic device depends on the geometry of the T-junction as well as the capillary number, flow ratio, and viscosity ratio of the two phases [11, 12]. However, this method is difficult to generate droplets with the size smaller than $10 \mu \mathrm{m}$ due to the large hydraulic diameter of the $\mathrm{T}$-junction structure due to the big surface tension from the long circumference. Wu et al. reported a pneumatic pressure driven $\mathrm{T}$-junction structure for producing droplets in microfluidic channel. The smallest droplet with the size of $13.9 \mu \mathrm{m}$ was formed [13].

This work proposed a simple and effective way to produce a micro-orifice structure to reduce the surface tension effect of the T-junction. The T-junction and the orifice structures 


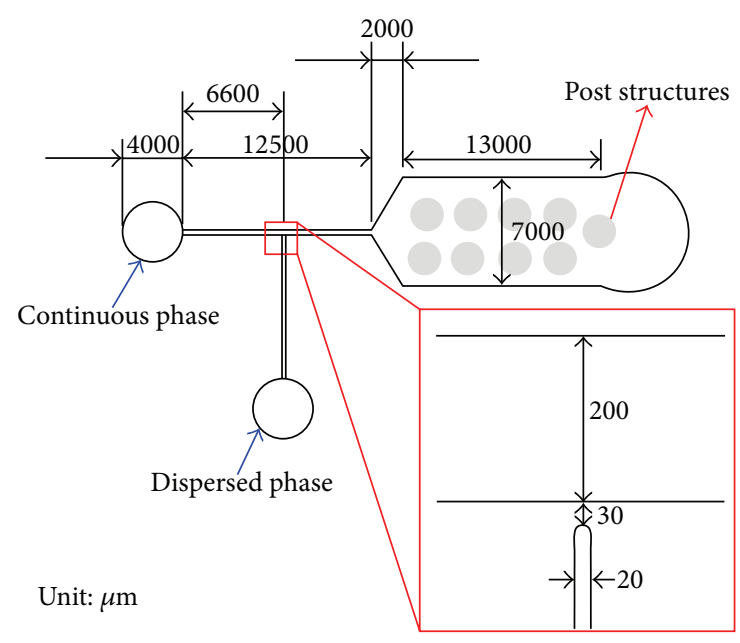

FIGURE 1: Photo mask design for the physical dimension of the microfluidic device for producing small water-based droplets.

are fabricated utilizing the isotropic undercut etching technique in a single etching process. The produced structure is then used to produce the water-based droplets via the micro-orifice. The relationship between the formed droplet size and the flow rate ratios of the two immiscible fluids is systematically investigated.

\section{Materials and Methods}

2.1. Chip Design. Figure 1 shows the mask design for the physical dimension of the proposed microfluidic device. Since a one-step isotropic wet etching technique is adopted to produce the micro-orifice at the T-junction, a gap of $30 \mu \mathrm{m}$ was designed between the main channel and the side channel. The target depth for the etching microfluidic channel is $15 \mu \mathrm{m}$, resulting in just etching through the designed gap, and forms a small etch-through hole beneath the etching mask. Note that a number of poststructures with the diameter of $300 \mu \mathrm{m}$ were designed at the downstream expansion channel to prevent the collapse of the channel during the thermal fusion bonding process.

2.2. Chip Fabrication. Figure 2 shows the simplified fabrication process for producing the microfluidic chip with the micro-orifice at the T-junction. The device was fabricated in low-cost soda-lime glasses with a rapid prototyping process. The details for the fabrication process can be found in our previous publication $[14,15]$. Briefly, commercial microscope slides were cleaned and patterned with a standard photolithography process. A thin layer of AZ4620 photoresist was used as the etching mask for etching microfluidic channels in BOE solution (Figures 2(a)-2(c)). Due to the reflective index difference between $\mathrm{BOE}$ solution and the glass substrate, the undercut etched region could be clearly observed using an optical microscope. The etching process was stopped while etching through the undercut region between the mail channel and side channel, resulting in an ultrasmall pore structure at the T-junction (Figure 2(d)). The etched substrate then stripped the photoresist etching mask and sealed with the thermal fusion bonding method at $580^{\circ} \mathrm{C}$ for $10 \mathrm{~min}$ (Figure 2(f)). Finally, the inlet and outlet ports were connected with Teflon tubing with the outer and inner diameters of $1.5 \mathrm{~mm}$ and $0.5 \mathrm{~mm}$, respectively.

Since the water-in-oil droplet formation system was used in the present study such that a hydrophobic layer was coated on the surface of the main channel to enhance the formation of water-based droplet. A thin layer of the organic-based spinon glass (SOG, 400F, Filmtronics, USA) was applied only on the main channel with the assistance of vacuum suction. The coated SOG layer was then cured at $400^{\circ} \mathrm{C}$ for $1 \mathrm{hr}$. in $\mathrm{N}_{2}$ atmosphere.

2.3. Experimental Setup. Figure 3 shows the experiment setup for producing and observing the formation of the water-in-oil droplets. Two immiscible liquids of dispersed phase and continuous phase (commercial olive oil) were injected into the microfluidic channels from the corresponding inlets using two syringe pumps (KDS 200, KD Scientific Inc., USA). Note that the employed continuous phase liquid was doped with $3 \%$ of Span 80 (Sigma, USA) as the surfactant to enhance the formation of droplets. For forming chitosan droplets, $1 \%$ of (by weight) aqueous chitosan solution (Sigma, USA) was used as the dispersed phase. Note that all the liquids were filtered with polymer filter with $0.2 \mu \mathrm{m}$ in size (Millipore, Ireland) prior to the test. An optical microscope (Eclipse TE2000-U, Nikon, Japan) equipped with a high speed digital camera (1200 fps, EX-F1, Casio, Japan) was used for observing the formation of droplets in the microchannel.

\section{Results and Discussions}

Figure 4 shows the scanning electron microscope (SEM) images of the undercut etched orifice structure at the Tjunction right after peeling the photoresist etching mask and after sealing. As described previously, the etching process could be easily observed using an optical microscope. In addition, the size of the etched orifice also could be precisely adjusted by controlling the etching time. It is clear that the undercut region formed a small notch at the T-junction (Figure 4(a)). Due to the limitation of the designed mask pattern $(20 \mu \mathrm{m}$ in resolution), the width of the undercut structure was around $10 \mu \mathrm{m}$ in width. This can be improved by using the photo mask with higher optical resolution. The dark region near the top glass substrate in Figure 4(b) presents the formed micro-orifice after sealing the channel. The image shows that the thermal fusion bonding did not collapse the micro-orifice structure and the depth for the orifice was around $1.5 \mu \mathrm{m}$. The calculated equivalent hydraulic diameter for this micro-orifice was around $2.4 \mu \mathrm{m}$. The smaller hydraulic diameter is beneficial for producing small droplets. Note that the orifice with smaller hydraulic diameter could also be formed by controlling the etching time.

In order to further improve the hydrophobicity of the produced microfluidic channel for droplet formation, a simple yet efficient permanent coating of organic-based spinon-glass layer (SOG 400F, Filmtronics, USA) was used to 


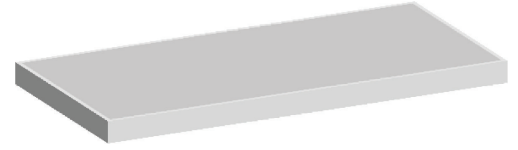

(a) Cleaning

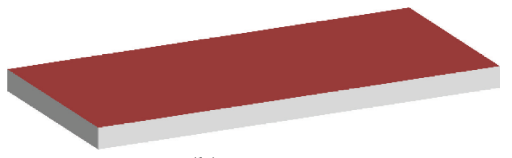

(b) PR spin coating

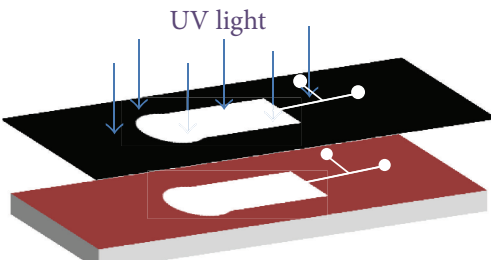

(c) Lithography

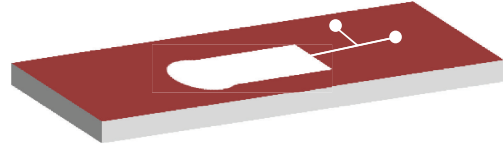

(d) Glass etching

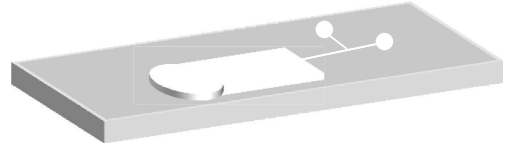

(e) PR stripping

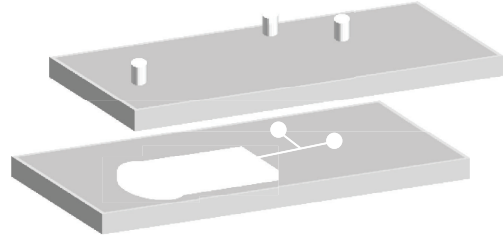

(f) Bonding

FIGURE 2: Schematic of the simplified fabrication process for the droplet-based microfluidic chip.

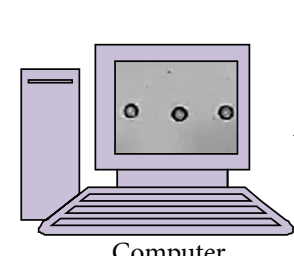

Computer

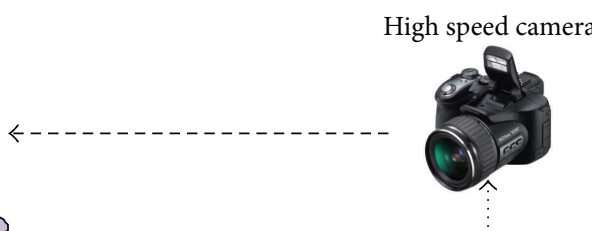

Filter

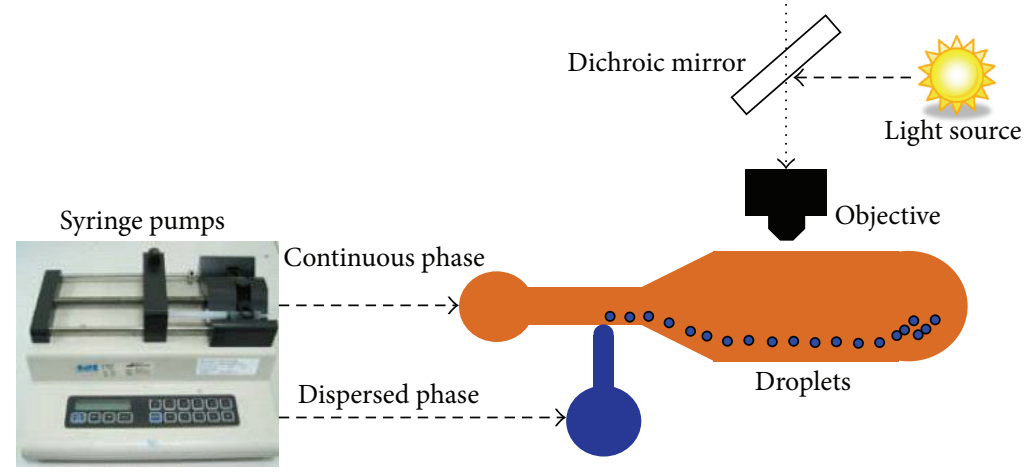

FIgURE 3: A schematic presentation of the experimental setup for droplet formation with the microfluidic chip with the ultrasmall orifice.

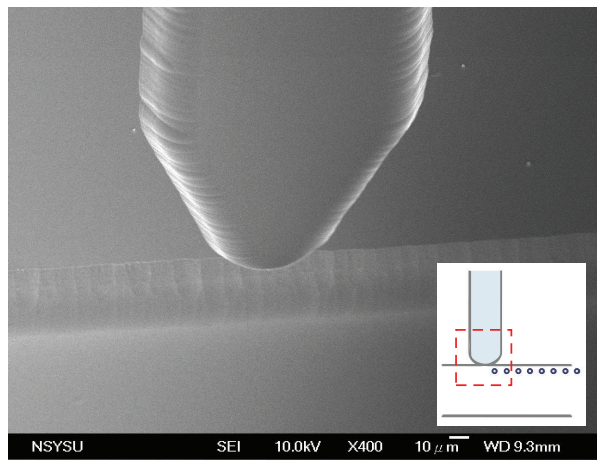

(a)

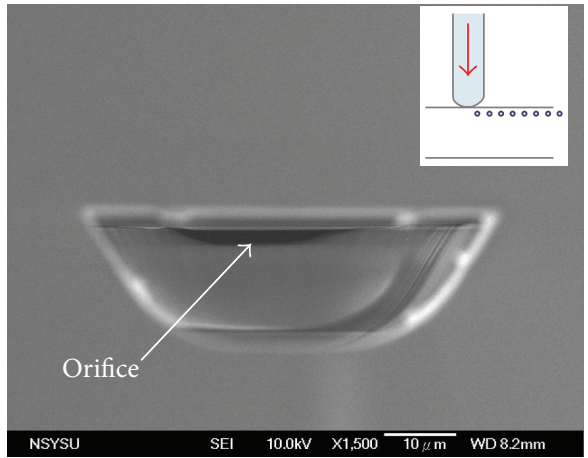

(b)

FIGURE 4: SEM images of the fabricated microorifices. (a) the undercut etched structure at the T-junction after pealing the PR; (b) the formed small orifice after thermal bonding. 


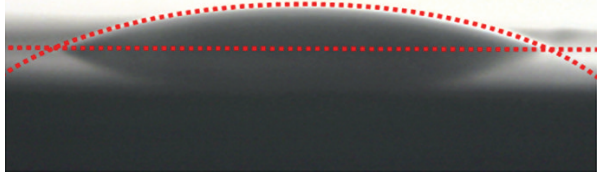

(a) $23.7^{\circ}$

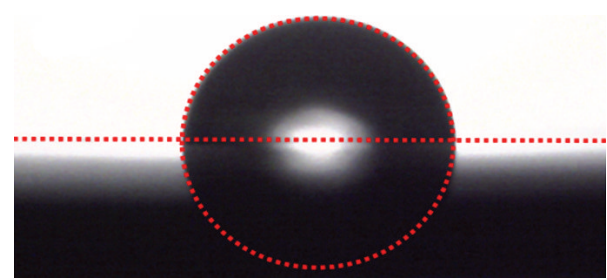

(b) $87.9^{\circ}$

FIGURE 5: The photo images showing the glass surface (a) without and (b) with the SOG coating layer.

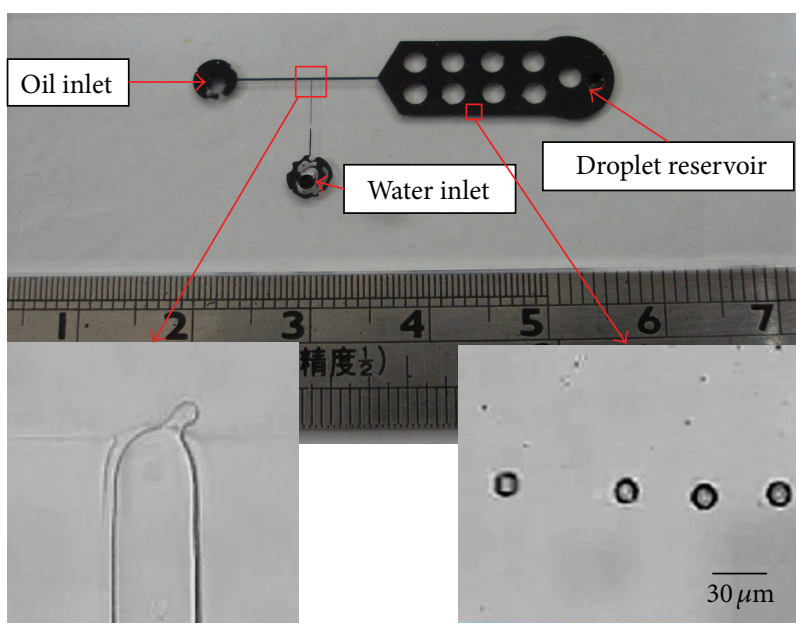

FIGURE 6: The photo image for the developed chip and the microscopic views for the droplet formation process.

modify the channel surface. Figure 5 presents the measured contact angles for a glass substrate without and with coating the organic-based SOG layer. Results showed that the SOG layer significantly increased the contact angle of water drop on the glass substrate. The photo images of the fabricated chip and the droplet formation process are given in Figure 6. The inset images at the bottom show that the dispersed fluid was squeezing out from the formed micro-orifice at the Tjunction and formed the small droplets downstream. The sheath flow in the main channel cut the squeezed fluid and formed the droplets by shearing force. Small droplets could be easily formed using the developed microfluidic chip with the micro-orifice.

The relationship between the diameter of the water droplets and the oil/water flow rate ratio is shown in Figure 7. The flow rate for the dispersed phase was set from $75 \mu \mathrm{L} / \mathrm{hr}$ to $125 \mu \mathrm{L} / \mathrm{hr}$. The equivalent hydraulic diameter for the orifice in this test was $1.5 \mu \mathrm{m}$. Results show that the higher flow rate ratio formed smaller droplets. Results also indicated that bigger droplets were formed at a slower water flow rate for the same flow rate ratio due to the grater shearing force for the faster flowing continuous phase. Compared to the two flow rate conditions of $125 \mu \mathrm{L} / \mathrm{hr}$ and $100 \mu \mathrm{L} / \mathrm{hr}$, the size for the formed droplets did not show significant difference. However, higher water flow rate required higher driving pressure to squeeze the dispersed fluid through the small orifice, resulting in the risk for damaging the orifice

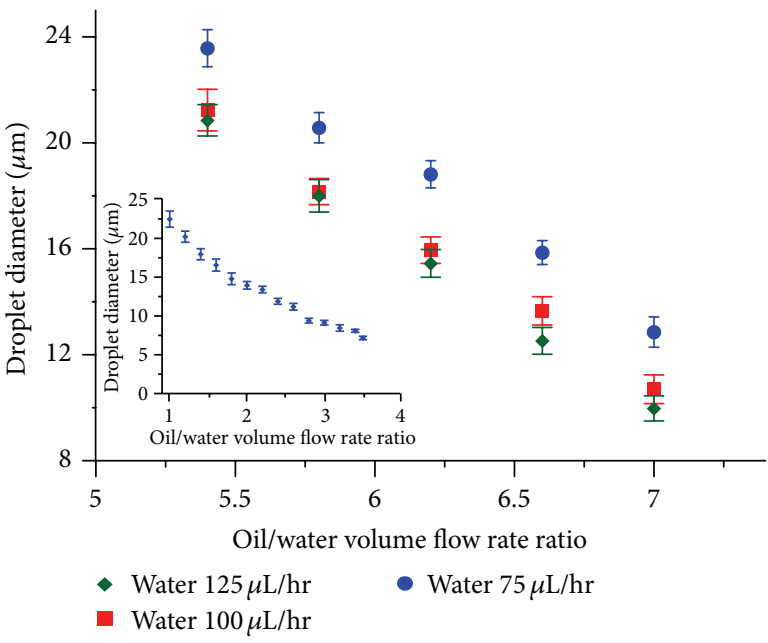

FIGURE 7: The relationship between the droplet size and the applied oil/water flow rate ratio while using the orifice of the equivalent hydraulic diameter of $1.5 \mu \mathrm{m}$. Note that the inset shows the same test using the orifice of the hydraulic diameter of $1.1 \mu \mathrm{m}$.

and tubing connection. Therefore, the dispersed phase flow rate for the following tests was set at $100 \mu \mathrm{L} / \mathrm{hr}$. The inset in Figure 7 presents the same size relationship while using a smaller orifice with the hydraulic diameter of $1.1 \mu \mathrm{m}$. Results show that droplets of the size smaller than $10 \mu \mathrm{m}$ could be produced for the flow rate ratio greater than 2.8. The smallest droplet size formed with this approach was $6.5 \mu \mathrm{m}$ at the flow rate ratio of 3.5, which was smaller than the reported droplet size formed with the chip having similar T-junction structure [13]. It is also noted that the flow rate ratio was difficult to be further increased since the increased driving pressure would cause the diameter expansion of the Teflon tubing and reduce the fluid pressure. This effect limited the smallest size of the formed droplets with this approach.

One of the issues for producing microdroplets via the small orifice is the great pressure drop at the orifice since the small orifice resulted in a small Reynolds number. In this regard, the flow at the orifice was stable such that the droplet production rate was only affected by the fluid flow rate in the side channel. Figure 8 shows the measured droplet production rate for the developed T-junction. Results showed that the droplet production rate was around 2 droplets per second while using the T-junction with the orifice of the equivalent hydraulic diameter of $1.1 \mu \mathrm{m}$. Note that the flow rate for the dispersed phase in this test was $100 \mu \mathrm{L} / \mathrm{hr}$. The size uniformity 


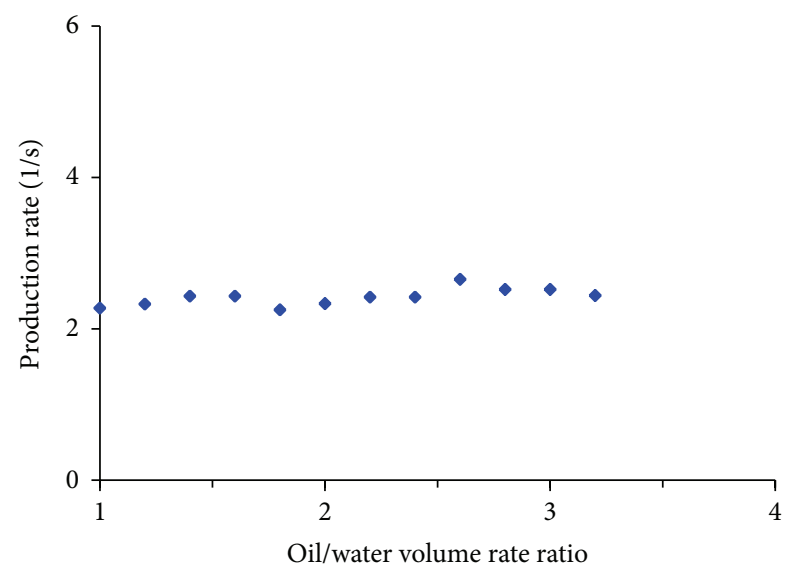

FIGURE 8: Measured production rate for ultrasmall droplets using the developed microfluidic device. Note that the equivalent hydraulic diameter for this case was $1.1 \mu \mathrm{m}$.

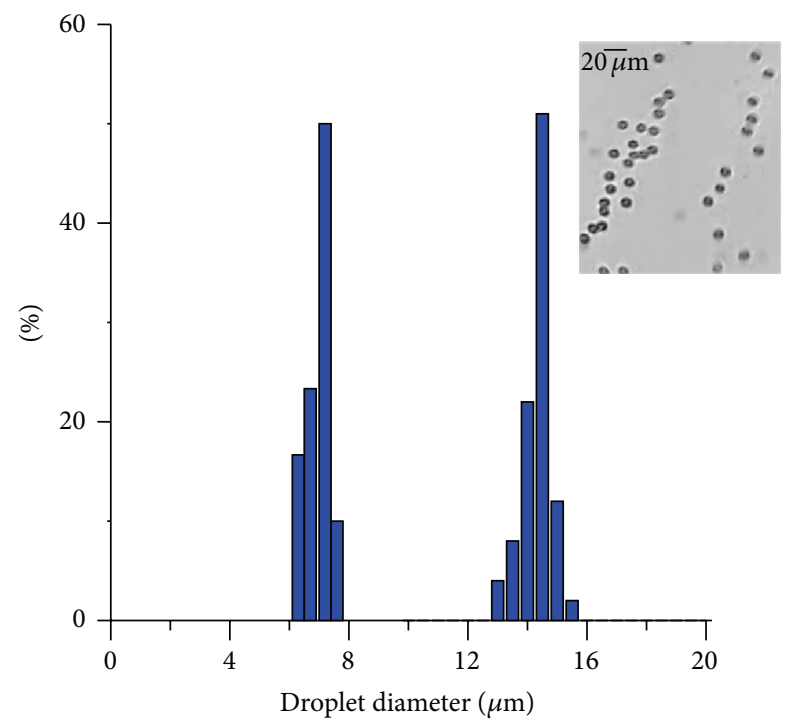

FIGURE 9: Droplet size distribution formed under the volume flow ratio of 2 and 3.5, showing the good uniformity for the formed droplets.

for the formed droplets is also an important issue for practical applications of the microfluidic device. The size distribution for the droplets formed at the flow rate ratios of 2 and 3.5 was analysed. The captured images of 150 individual droplets were analysed using the digital image processing technique. Figure 9 shows the measured size distribution for the droplets formed with these two conditions. The statistical results show that the average diameters were $13.5 \pm 0.47 \mu \mathrm{m}$ and $6.9 \pm$ $0.40 \mu \mathrm{m}$ for these two cases. The calculated corresponding coefficient of variation (CV) was $3.13 \%$ and $5.7 \%$, respectively. Results indicated that the generated droplets exhibited good size uniformity.

Chitosan is a linear polymer and has a number of potential biomedical applications, especially for drug encapsulation in pharmaceutical applications. The viscosity of chitosan at room temperature is around $5-10 \mathrm{cP}$ which is much higher

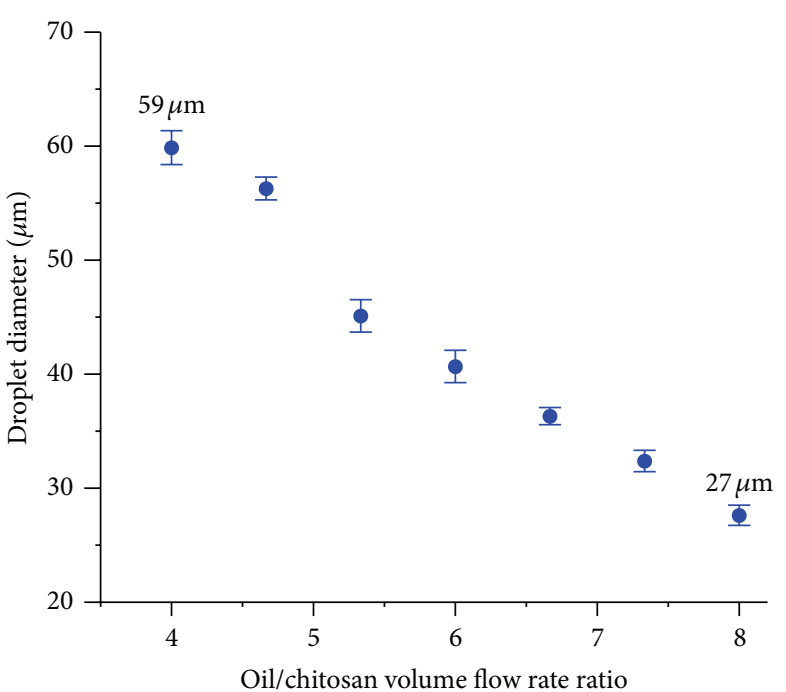

FIGURE 10: The relationship between the diameter of the chitosan droplets and the flow rate ratio. Note that the orifice used in this test was with the equivalent hydraulic diameter of $21.2 \mu \mathrm{m}$.

than the viscosity of water at the same condition. Therefore, the $\mathrm{T}$-junction structure with a bigger orifice was required to produce chitosan droplets. Figure 10 shows the relationship between the size of formed chitosan droplets and the applied flow rate ratio. The equivalent hydraulic diameter for the orifice at the T-junction was $21.2 \mu \mathrm{m}$. Results show that chitosan droplets of the size ranging from 27 to $59 \mu \mathrm{m}$ could be successfully produced, which were much smaller than the chitosan droplets formed with similar hydrodynamic approaches $[16,17]$.

\section{Conclusions}

This study reported a novel undercut etching method to produce a T-junction structure with microorifices using a single wet-etching process. Microdroplets of small sizes could be easily generated via the orifice with smaller hydraulic diameters. Results showed that the developed device could successfully produce uniform water-based droplets in olive oil with the size of smaller than $10 \mu \mathrm{m}$. The droplet production rate kept stable with the oil/water flow rate ratio in the range of 1 to 3.6 while using the T-junction with the orifice of the equivalent hydraulic diameter of $1.1 \mu \mathrm{m}$. In addition, chitosan droplets of the size as small as $27 \mu \mathrm{m}$ could also be produced with the orifice of the hydraulic diameter of $21.2 \mu \mathrm{m}$. The present study provides a simple yet reliable way to produce small droplets in microfluidic systems.

\section{Acknowledgment}

The financial support from the National Science Council of Taiwan is greatly acknowledged.

\section{References}

[1] M. T. Guo, A. Rotem, J. A. Heyman, and D. A. Weitz, "Droplet microfluidics for high-throughput biological assays," Lab on a Chip, vol. 12, no. 12, pp. 2146-2155, 2012. 
[2] D. J. Harrison, K. Fluri, K. Seiler, Z. Fan, C. S. Effenhauser, and A. Manz, "Micromachining a miniaturized capillary electrophoresis-based chemical analysis system on a chip," Science, vol. 261, no. 5123, pp. 895-897, 1993.

[3] T. Glawdel, C. Elbuken, and C. L. Ren, "Droplet formation in microfluidic T-junction generators operating in the transitional regime. I. Experimental observations," Physical Review E, vol. 85, no. 1, Article ID 016322, 2012.

[4] P. Garstecki, M. J. Fuerstman, H. A. Stone, and G. M. Whitesides, "Formation of droplets and bubbles in a microfluidic Tjunction-scaling and mechanism of break-up," Lab on a Chip, vol. 6, no. 3, pp. 437-446, 2006.

[5] J. H. Xu, S. W. Li, J. Tán, Y. J. Wang, and G. S. Luo, "Preparation of highly monodisperse droplet in a T-junction microfluidic device," AIChE Journal, vol. 52, no. 9, pp. 3005-3010, 2006.

[6] J. H. Xu, S. W. Li, J. Tan, and G. S. Luo, "Correlations of droplet formation in T-junction microfluidic devices: from squeezing to dripping," Microfluidics and Nanofluidics, vol. 5, no. 6, pp. 711717, 2008.

[7] C. H. Lan, S. W. Lin, and C. H. Lin, "Microfluidic T-junction with an undercut orifice to generate ultra-small droplets," in Proceedings of the 5th Asia-Pacific Conference on Transducers and Micro-Nano Technology (APCOT '10), Perth, Australia, 2010.

[8] C. Cramer, P. Fischer, and E. J. Windhab, "Drop formation in a co-flowing ambient fluid," Chemical Engineering Science, vol. 59, no. 15, pp. 3045-3058, 2004.

[9] G. G. Su, P. W. Longest, and R. M. Pidaparti, "A novel micropump droplet generator for aerosol drug delivery: design simulations," Biomicrofluidics, vol. 4, no. 4, Article ID 044108, 2010.

[10] S.-K. Hsiung, C.-T. Chen, and G.-B. Lee, "Micro-droplet formation utilizing microfluidic flow focusing and controllable moving-wall chopping techniques," Journal of Micromechanics and Microengineering, vol. 16, no. 11, article 022, pp. 2403-2410, 2006.

[11] T. Glawdel and C. L. Ren, "Droplet formation in microfluidic T-junction generators operating in the transitional regime. III. Dynamic surfactant effects," Physical Review E, vol. 86, no. 2, Article ID 026308, 2012.

[12] C.-H. Lin, L.-M. Fu, and Y.-S. Chien, "Microfluidic T-form mixer utilizing switching electroosmotic flow," Analytical Chemistry, vol. 76, no. 18, pp. 5265-5272, 2004.

[13] H.-W. Wu, Y.-C. Huang, C.-L. Wu, and G.-B. Lee, "Exploitation of a microfluidic device capable of generating size-tunable droplets for gene delivery," Microfluidics and Nanofluidics, vol. 7, no. 1, pp. 45-56, 2009.

[14] C.-H. Lin, C.-H. Tsai, and L.-M. Fu, "A rapid three-dimensional vortex micromixer utilizing self-rotation effects under low Reynolds number conditions," Journal of Micromechanics and Microengineering, vol. 15, no. 5, pp. 935-943, 2005.

[15] C.-H. Lin, G.-B. Lee, Y.-H. Lin, and G.-L. Chang, "A fast prototyping process for fabrication of microfluidic systems on sodalime glass," Journal of Micromechanics and Microengineering, vol. 11, no. 6, pp. 726-732, 2001.

[16] D. Mark, S. Haeberle, R. Zengerle, J. Ducree, and G. T. Vladisavljević, "Manufacture of chitosan microbeads using centrifugally driven flow of gel-forming solutions through a polymeric micronozzle," Journal of Colloid and Interface Science, vol. 336, no. 2, pp. 634-641, 2009.

[17] C.-H. Yang, Y.-S. Lin, K.-S. Huang et al., "Microfluidic emulsification and sorting assisted preparation of monodisperse chitosan microparticles," Lab on a Chip, vol. 9, no. 1, pp. 145150, 2009. 

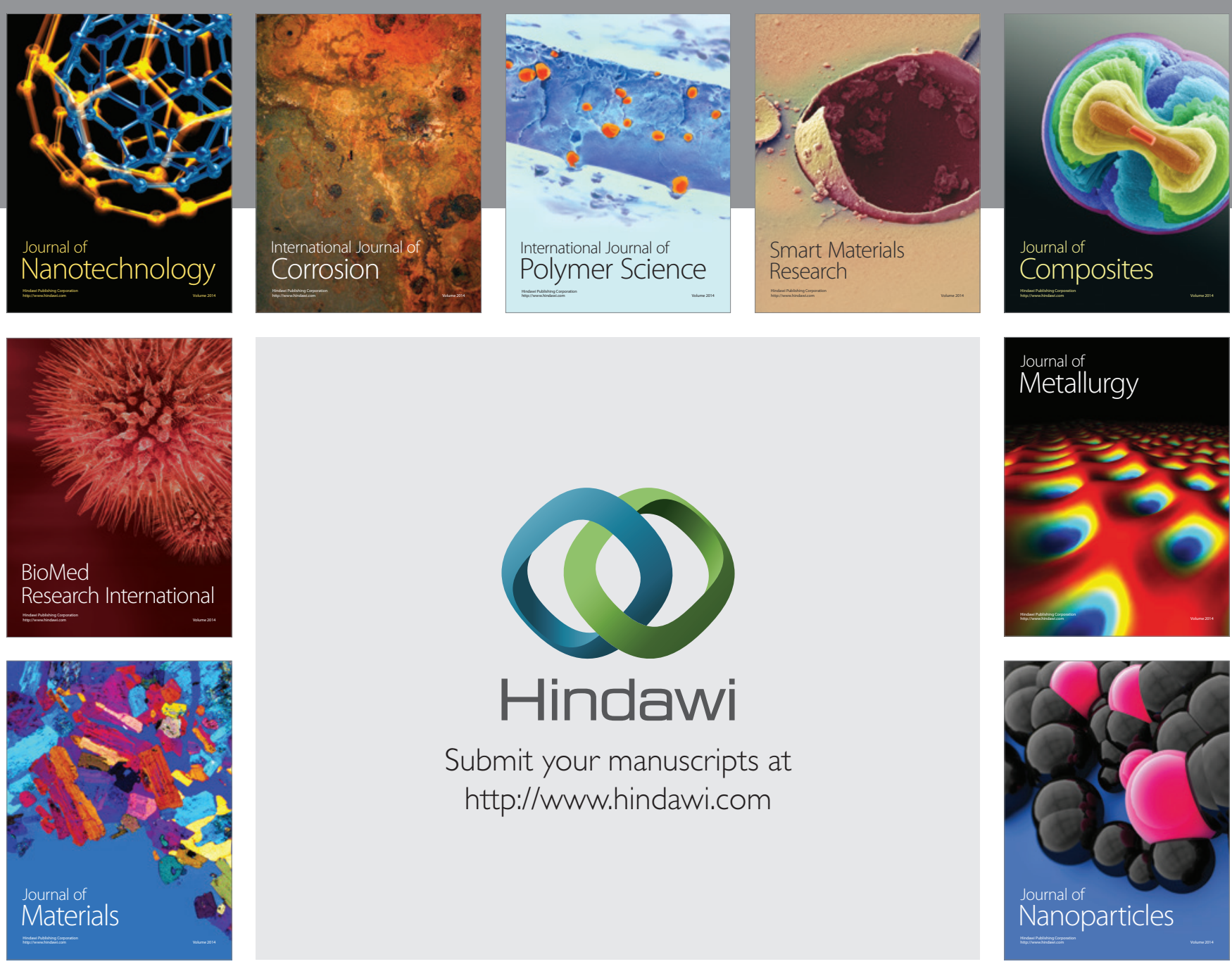

Submit your manuscripts at http://www.hindawi.com
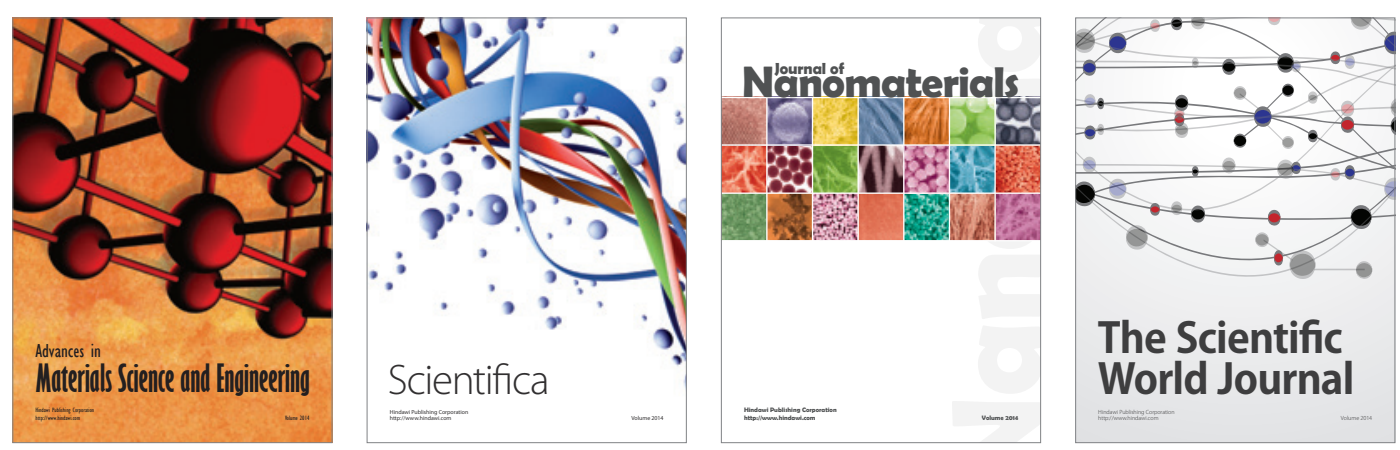

\section{The Scientific World Journal}
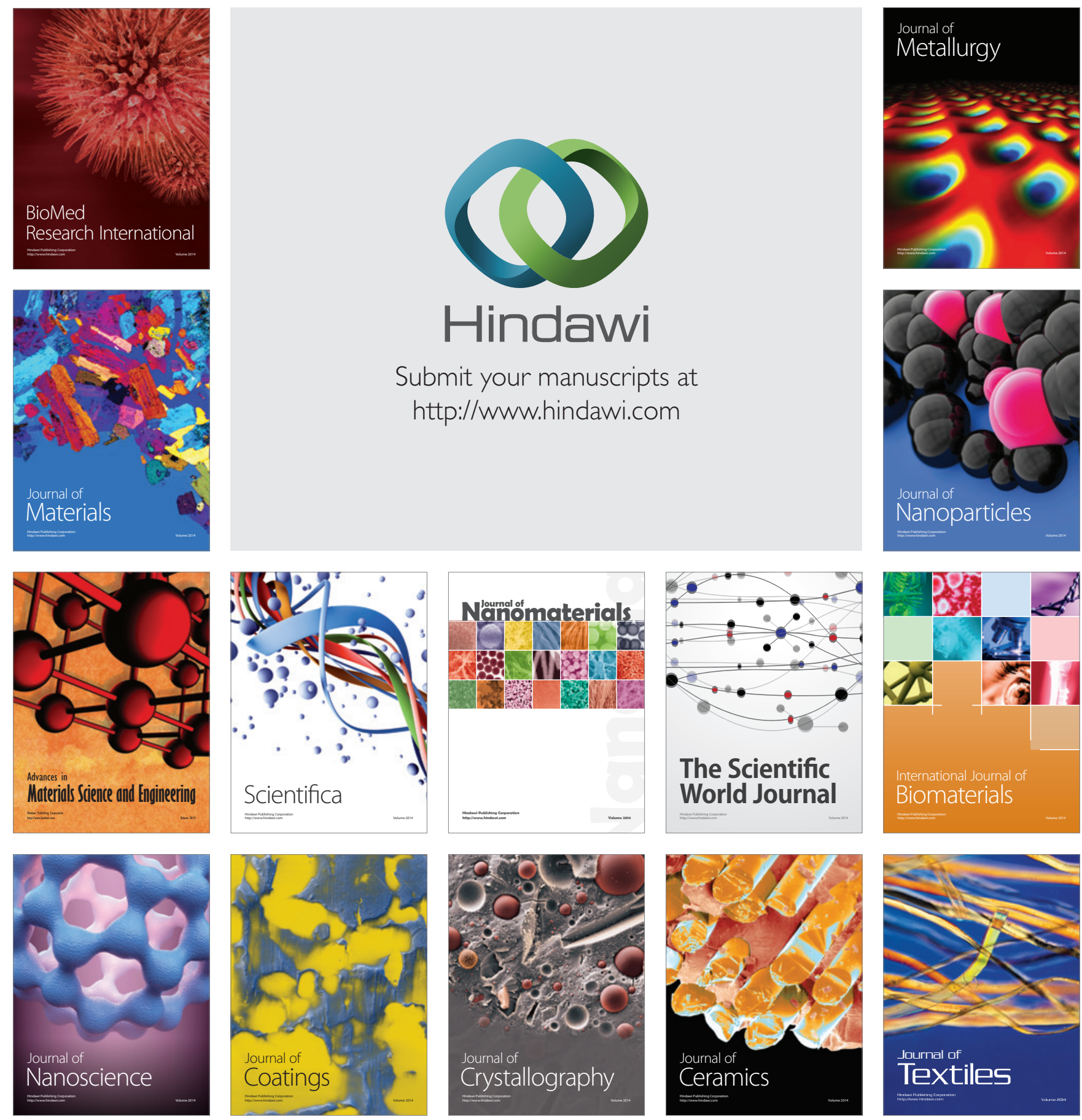